\title{
Produktionsstrategien im Bereich der chemischen Wirkstoffherstellung unter Berücksichtigung finanzwirtschaftlicher Aspekte
}

\author{
Hermann Wiederkehr*
}

\section{1. Übersicht}

Auf dem Gebiet der Fertigung werden laufend neue, sich zum Teil widersprechende, Produktionsphilosophien angepriesen. Alle diese Strategien versprechen eine hohe Wirtschaftlichkeit, d.h. die Bereitstellung der Verkaufsprodukte zu den für das Unternehmen tiefst möglichen Kosten. Erwähnt seien folgende, momentan im Vordergrund stehende Produktionsstrategien:

- Just-in-Time Produktion (JIT)

und

- die durchgehende 7-Tage-Produktion (hohe Auslastung)

Die erstere verspricht tiefe Gestehungskosten, da vor allem die 'Lagerkosten', d.h. die Kosten für das in den Lagerbeständen gebundene Kapital, klein gehalten werden können, die zweite, da die Produktionsanlagen, welche hohe Investitionen erfordern, optimal genutzt werden.

In der Praxis ist es aber nicht möglich, gleichzeitig beide Anforderungen zu erfüllen. Es besteht ein Widerspruch zwischen der Forderung nach flexibler Produktion (JIT) und der gleichzeitigen Forderung nach einem hohen Auslastungsgrad der Anlagen und des Personals.

Im folgenden soll versucht werden, diese Problematik am Beispiel der chemischen Wirkstoffherstellung aus einer möglichst ganzheitlichen Sicht zu betrachten. Zu diesem Zweck ist es notwendig, sich einerseits über die unternehmerischen Ziele und andererseits sowohl über die produktionsspezifischen, wie auch über die finanzwirtschaftlichen Zusammenhänge im Unternehmen Klarheit zu verschaffen.

\section{Zielsetzungen im Unternehmen}

Im heutigen wettbewerbsorientierten Umfeld kommt der Ausrichtung des unternehmerischen Handelns auf den Kunden vorrangige Bedeutung zu. An oberster Stelle steht die Befriedigung des Kunden.
Auf die Produktionsstrategie übertragen bedeutet die geforderte Kundenausrichtung, die Gewährleistung einer hohen Lieferbereitschaft im Unternehmen.

Neben der Kundenorientierung spielen die Kosten eine wichtige Rolle. Längerfristig kann ein Unternehmen nur existieren, wenn die Kosten stimmen. Man spricht in diesem Zusammenhang von der Dominanz der Kosten. Das längerfristige Ziel der Unternehmung muss (unter anderem) die wirtschaftliche Selbsterhaltung sein. In bezug auf die im Unternehmen anzuwendende Produktionstrategie resultiert aus den finanzwirtschaftlichen Zielsetzungen die Forderung nach tiefen Produktebereitstellungskosten.

Verlangt werden also vom heutigen Unternehmen demnach:

- Eine hohe Lieferbereitschaft (Kundenausrichtung) und

- die Bereitstellung der Produkte zu tiefen Kosten (Dominanz der Kosten)

\section{Aufzeigen der Zusammenhänge}

\subsection{Produktionsspezifische Aspekte}

3.1.1. Strukturen

Die Produktion im Unternehmen ist gekoppelt mit:

- Verkauf

- Materialbewirtschaftung (Lager)

- Personal

- Anlagen usw.

Eine Möglichkeit, komplexe Zusammenhänge darzustellen, wird von den Professoren Ulrich und Probst im Buch 'Ganzheitliches Denken' (Verlag Paul Haupt, 1988) beschrieben. In einem sogenannten Vernetzungsmodell werden alle möglichen Beziehungen und Verknüpfungen mit Pfeilen dargestellt. Die Pfeile werden je nach Art der Beziehung mit einem (+) oder (-) Zeichen versehen. Eine Beziehung mit einem $(+)$ Zeichen bedeutet, dass sich bei einer Änderung in der Ausgangsgrösse die Bezugsgrösse in der gleichen Richtung ändert und bei einer Beziehung mit einem $(-)$

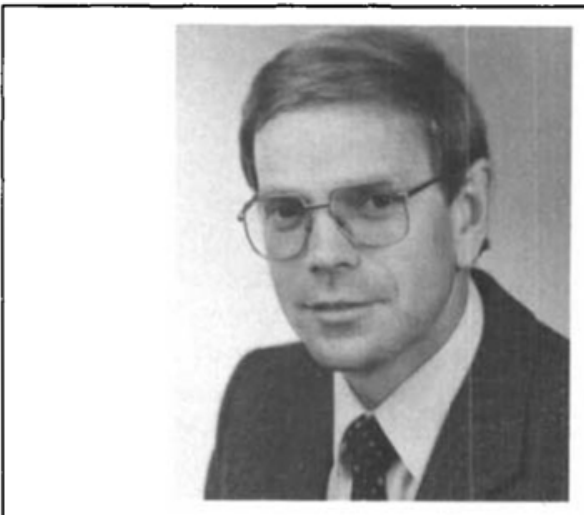

Hermann Wiederkehr: Geboren 1942 in Bünzen, Kanton Aargau. 1963-1967 Chemiestudium an der Eidgenössischen Technischen Hochschule (ETH), Zürich, Ausbildungsrichtung Chemie-Ingenieur; 1970 Promotion bei Prof. Dr. W. Richarz. Eintritt in die Firma F. Hoffmann-La Roche AG, Basel, im selben Jahr. 1970-1973 Schottlandaufenthalt: Inbetriebnahme einer Produktionsanlage. 1973-1981 Technisch-chemische Entwicklung; 1977 Ernennung zum wissenschaftlichen Spezialisten, $1982 \mathrm{Be}$ förderung zum Prokuristen. 1982-1987 Leitung einer Gruppe chemischer Produktionsbetriebe; 1988 Beförderung zum Vizedirektor und Úbertritt in den Stab Bereich Produktion Konzern. 1976-1987 Delegierter der Schweizerischen Gesellschaft für Chemische Industrie (SGCI) in der Fachgruppe für Technische Kristallisation der Europäischen Föderation für Chemie-Ingenieurwesen (EFCE).

Zeichen entsprechend in der entgegengesetzten Richtung.

In Fig. 3 ist ein einfaches Modell für die Produktion dargestellt. Das Modell liest sich z.B. wie folgt (dynamische Betrachtung):

Frage: Was passiert, wenn die Verkäufe steigen?

- Als Folge der Steigerung der Verkäufe sinkt der Bestand im Lager (entgegengerichtete Beziehung: steigt--sinkt).

- Wenn der Bestand im Lager sinkt, wird folglich die Produktion gesteigert (entgegengerichtete Beziehung: sinktsteigt).

- Durch die Erhöhung der Produktion nimmt der Bestand im Fertiglager zu (gleichgerichtete Beziehung: steigtsteigt).

- Wie man sieht, wird im Modell die 'Störung', welche vom Verkauf ausgegangen ist, durch die logischen Verknüpfungen zwischen Lager und Produktion ausgeglichen. Man spricht in diesem Fall von einer stabilisierenden bzw. ausgleichenden Kreislaufbeziehung zwischen Lager und Produktion. Ob eine Kreislaufbeziehung stabilisierend oder destabilisierend bzw. aufschaukelnd wirkt, ergibt sich aus der Multiplikation der Vorzeichen: $(-)$ stabilisierende Kreislaufbezie-

* Korrespondenz: Dr. H. Wiederkehr

F. Hoffmann-La Roche $A G$

Werk Lalden

CH-3931 Lalden/VS 
hung, (+) aufschaukelnde Kreislaufbeziehung.

- Aus dem Modell ist auch ersichtlich, dass bei einer Steigerung der Produktion mehr Anlagen und mehr Personal benötigt wird.

In Fig. 4 ist ein verfeinertes Vernetzungsmodell für die Produktion dargestellt. Das Grundmodell (Fig. 3) wurde durch Hinzufügen von weiteren Abhängigkeiten und Beziehungen erweitert. Das Modell erlaubt die Diskussion verschiedener Fragestellungen ausgehend von einem beliebigen Punkt.

\subsubsection{Produktionsstrategien}

Bei der Beantwortung der Frage nach der Produktionsstrategie muss man sich klar werden über die Anforderung an die Produktion im Rahmen der Produktebereitstellung im Unternehmen. Wie wir gesehen haben, steht die Gewährleistung einer hohen Lieferbereitschaft zu tiefen Produktebereitstellungskosten im Vordergrund. Da die Bedarfe an den Verkaufsprodukten aber den Marktschwankungen unterliegen und somit keineswegs stetig anfallen, muss die Gewährleistung der Lieferbereitschaft auch unter diesen erschwerenden Bedingungen erfüllt werden.

Die Anforderung an die Produktion lautet somit: Gewährleistung einer hohen Lieferbereitschaft bei schwankenden Bedarfen zu minimalen Produktebereitstellungskosten.

Die Erschwernis, dass die Lieferbereitschaft auch bei stark schwankenden Bedarfen sichergestellt werden muss, bedingt zusätzlich die Forderung nach Flexibilität im Bereich der Bereitstellung der Produkte. Die Flexibilität kann im Unternehmen auf verschiedene Arten gewährleistet werden. Zum Auffangen der Bedarfsschwankungen, welche vom Verkauf herrühren, bieten sich folgende Strategien an:

I. Auffangen der Bedarfsschwankungen seitens des Verkaufs durch Lagerbestände (Flexibilität durch Lager) und/oder

II. Durch Flexibilität im Bereich der Herstellung (Herstellen auf Abruf, JIT)

\begin{tabular}{|l|}
\hline Ziel: \\
- hohe Auslastung \\
- Economy of scale \\
\\
Charakteristika: \\
- Bewirtschaftung der Anlagen und des \\
- Personals \\
- (wene Kapazitätsauslastung Anlagen, wenig Personal) \\
- grosse Lose \\
(kleiner Anteil an Rüst- u.Umstellzeit) \\
- Vermeidung von "Stop and go" \\
- Abdeckung der Bedartsschwankungen aus \\
Lagerbeständen
\end{tabular}

Fig. 1. Traditionelle Produktionsphilosophie
Die erste Möglichkeit entspricht der traditionellen Produktionsphilosophie, bei welcher das Hauptgewicht auf einer hohen Auslastung der Anlagen liegt (Fig. 1). Heute modern scheint aber vor allem die zweite Strategie zu sein. Diesen Eindruck bekommt man wenigstens, wenn man einen Blick in die Fachzeitschriften wirft Den bekanntesten Vertreter dieser Strategie findet man unter der schon erwähnten Bezeichnung 'Just-in-Time Produktion' (Fig. 2) . Die JIT-Produktionsphilosophie wird als eine Möglichkeit angesehen, um sowohl die Flexibilität zu erhöhen, als auch die Lagerbestände zu senken und trotzdem eine hohe Lieferbereitschaft zu gewährleisten. Die Frage stellt sich nur, zu welchen Kosten (vgl. Beispiele).

\subsubsection{Darstellung der Produktionsstrate-} gien im Vernetzungsmodell

Man kann nun versuchen, im Produktionsvernetzungsmodell (Fig. 4) die Produktionsstrategien einzubeziehen. Wir haben gesehen, dass zur Gewährleistung der Lieferbereitschaft die Flexibilität eine zentrale Rolle spielt. In der klassischen Produktionsart werden die Bedarfsschwankungen aus Lagerbeständen aufgefangen (Flexibilität durch Lager), während nach der modernen Produktionsphilosophie keine Lager gehalten werden, sondern auf Abruf produziert wird. Dies bedingt, dass man grosse Flexibilität im Bereich der Herstellung besitzen muss (Flexibilität durch Produktion)

In der Darstellung Fig. 8 ist das Produktionsvernetzungsmodell durch die entsprechenden produktionsstrategischen Elemente erweitert worden. Gelb eingetragen sind die Auswirkungen, welche sich im Falle der Anwendung der traditionellen Produktionsstrategie ergeben und grün jene, welche sich ergeben, wenn die JITProduktionsstrategie praktiziert wird.

Aus der Darstellung geht hervor, dass in beiden Fällen der Einbau von Flexibilität die Bereitstellung von Reserven bedingt, im ersteren in Form von Lagern und im zweiten in Form von Personal, Anlagen und Rohstoffen. Ebenfalls ersichtlich ist, dass die Einführung von Flexibilität soge-

Produktion nach Bedarf (auf Abruf)
$==>$ Fertigung "Just in Time"
Ziel:
Bestände vermeiden
Charakteristiken:
- Flexibilität im Bereich der Fertigung
- Minimale Durchlaufzeiten (Prozessintegration,
Parallel-produktion, minimale Rüstzeiten)
- kleine Losgrössen
- Einbettung der Zulieferanten
- CIM (MRP-, PPS-Systeme usw)

Fig. 2. JIT-Fertigung nannte aufschaukelnde Kreislaufbeziehungen zur Folge hat. Im Falle der JIT-Strategie sind es sogar deren drei solcher destabilisierender Kreislaufbeziehungen, nämlich:

\section{Produktion - Personal \\ Produktion - Anlagen \\ Produktion - Rohstoffe}

Während bei der Anwendung der traditionellen Produktionsstrategie nur eine aufschaukelnde Wechselbeziehung im Bereich des Lagers zur Wirkung kommt.

Nachdem die produktionsspezifischen Zusammenhänge erläutert worden sind, interessiert uns die Frage, welche Produktionsstrategie im Unternehmen angewandt werden soll. Die Beantwortung dieser Frage kann nicht vorgenommen werden, ohne die Auswirkungen der praktizierten Produktionsstrategie auf die Kosten im Unternehmen zu berücksichtigen. Deshalb soll kurz auf die finanzwirtschaftlichen $\mathrm{Zu}$ sammenhänge eingegangen werden

\subsection{Finanzwirtschaftliche Aspekte}

Wie wir schon festgestellt haben, ist das Ziel jeder Unternehmung die wirtschaftliche Selbsterhaltung. Dies bedeutet, dass das Unternehmen einen finanziellen Ertrag erwirtschaften muss. Dieser Ertrag ist erforderlich, um einerseits die laufenden Betriebskosten zu decken und um andererseits die notwendigen Abschreibungen auf den Anlagen vornehmen zu können, damit die erforderlichen Mittel für deren Erneuerung bzw. späteren Einsatz bereitgestellt werden können. Zudem muss aus dem erwirtschafteten Ertrag eine angemessene Verzinsung des im Unternehmen investierten Kapitals resultieren. Die Messgrösse für den finanziellen Erfolg des Unternehmens ist der Return on Investment (ROI).

$$
\mathrm{ROI}=\frac{\text { Gewinn }}{\text { Anlagevermögen }+ \text { Umlaufvermögen }}
$$

Von Bedeutung für unsere Betrachtungen ist auch die Ưberlegung, wie im Unternehmen die finanziellen Mittel gebunden bzw. freigesetzt werden. Man spricht in diesem Zusammenhang vom betrieblichen Kreislauf der finanziellen Mittel. Bevor man produzieren kann, müssen die Anlagen angeschafft werden (Ausgaben für die Anlagen). Während der Herstellung des Produkts werden weitere Mittel benötigt für den Kauf von Rohstoffen, Hilfsmittel, die Entlöhnung des Personals usw. Erst wenn die Produkte verkauft werden, fliesst Geld zurück in die Unternehmung. Während des ganzen Herstellungsprozesses sind die finanziellen Mittel blockiert und verursachen in Form von Zinsen, $\mathrm{Ab}$ schreibungen usw. Kosten. Erst mit dem Verkauf wird Geld freigesetzt. Aus diesen Zusammenhängen ergibt sich für die Unternehmung eine doppelte Zielsetzung. Als erstes muss versucht werden, mit möglichst wenig Kapital (Anlagevermögen, Umlaufvermögen) die Produkte herzustellen $-\mathrm{Mi}$ - 

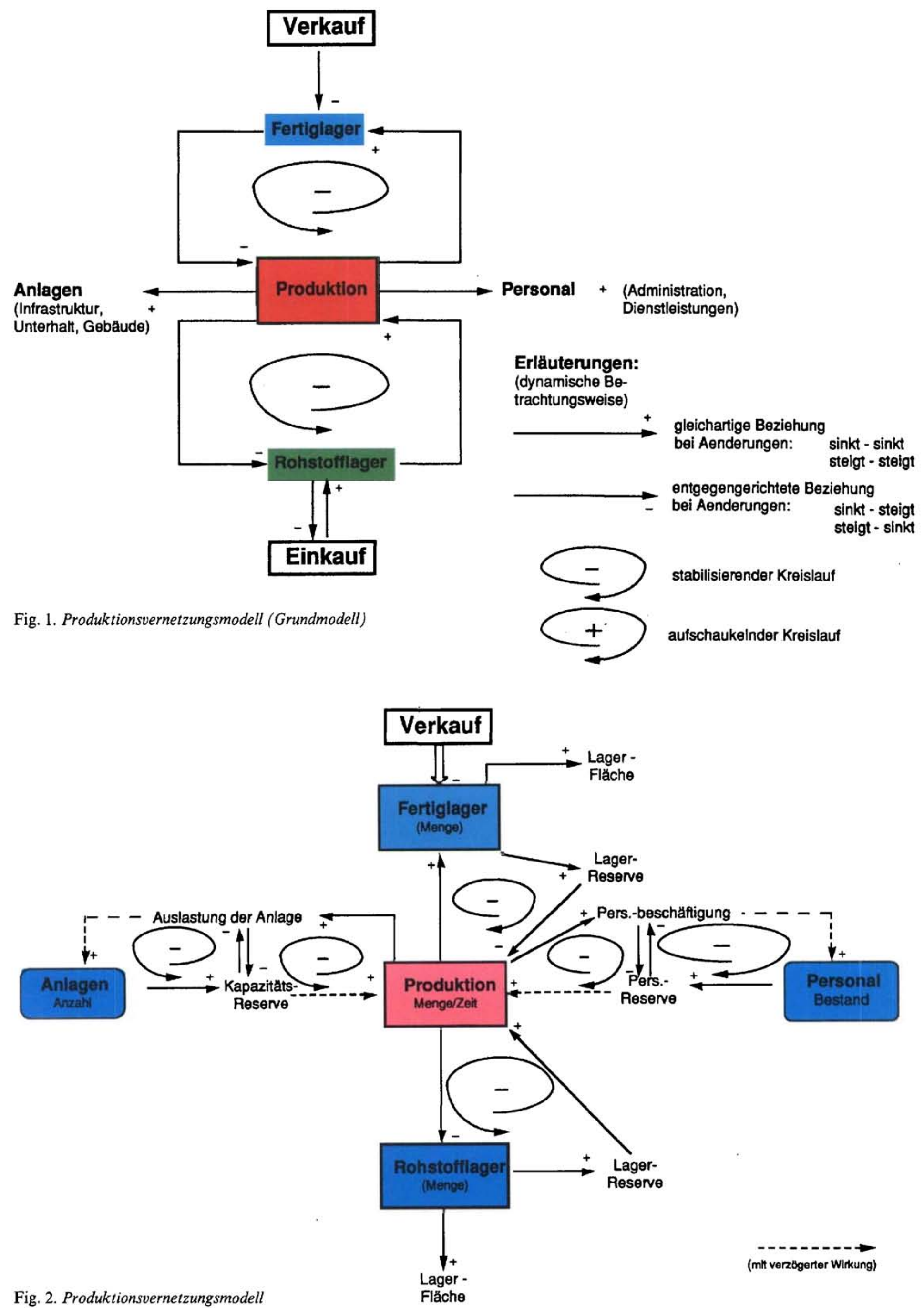
nimierung des Kapitaleinsatztes für die Leistungserstellung - und zweitens muss versucht werden, den Zeitraum von der Herstellung bis zum Verkauf möglichst kurz zu halten, damit das gebundene Kapital so schnell wie möglich freigesetzt wird (hohe Umsetzungsfrequenz, kurze Durchlaufzeit).

Die 'Produktbereitstellungskosten', welche für eine Unternehmung anfallen, setzen sich im wesentlichen zusammen aus:

\section{Herstellungskosten \\ - Materialkosten \\ - Personalkosten \\ - Kosten für Energie, Unterhalt, \\ Entsorgung, Qualitätssicherung usw.}

und

2. Kapitalkosten für:

- Produktionsanlagen

(Abschreibungen für Ersatz, Kosten

für das in den Anlagen gebundene

Kapital 'Anlagevermögen') [1]

- Lagerhaltung

(Gebäude, Einrichtungen, Kosten für das in den Produkten und Rohstoffen gebundene Kapital 'Umlaufvermögen')

Für die Leistungserstellung einer Unternehmung müssen neben den eigentlichen Herstellungskosten auch die Aufwendungen für die zur Leistungserstellung notwendigen Kapitalkosten für Anlagen und Materialien berücksichtigt werden. In den Diskussionen über Produktionsstrategien wird der Aspekt der Kosten für die Produktionsanlagen meistens nicht berücksichtigt. Die Beachtung aber gerade dieses Aspekts ist, wie wir später noch sehen werden, bei produktionsstrategischen Überlegungen von grosser Bedeutung.

Für unsere Überlegungen ist es zweckmässig, die Produktionskosten aufzuteilen in:

- 'operationelle Kosten' (Herstellungskosten bzw. variable Kosten)

und

- 'strategische Kosten' (Kapitalkosten, fixe Kosten)

Im folgenden beschränken wir uns auf die sogenannten strategischen Kosten. Es sind dies die Kosten, welche primär durch die im Unternehmen praktizierte Produktionsstrategie bestimmt werden.

Aus finanzwirtschaftlicher Sicht ergeben sich zwei Ansatzpunkte, die strategischen Kosten tief zu halten:

I. Optimierung des 'Anlagevermögens' (wenig Anlagen - hohe Auslastung)

und/oder

II. Optimierung des 'Umlaufvermögens' (kleine Lager - flexible Fertigung, JIT)

\section{Wahl der Produktionsstrategie}

Welche Strategie im Einzelfall die wirtschaftliche und damit die erfolgversprechende Produktionsstrategie ist, muss für jedes Unternehmen im einzelnen abgeklärt werden. $\mathrm{Zu}$ diesem Zweck ist eine möglichst ganzheitliche Betrachtung erforderlich. Es müssen sowohl die produktionsspezifischen wie auch die finanzwirtschaftlichen Aspekte mit einbezogen werden.

\section{'variable Kosten'}

'fixe oder strategische Kosten'

Ausschlaggebend für die Wahl der Produktionsstrategie werden schlussendlich die für die Leistungserstellung im Unternehmen erforderlichen Kosten sein.

Ein Hilfsmittel bei der Beantwortung solcher Fragen kann das in Fig. 9 dargestellte 'Integrale Produktionsvernetzungsmodell' sein. Das in Kap. 3.1 entwickelte Vernetzungsmodell (Fig. 8) wurde um die in Kap. 3.2 diskutierten Kostenelemente erweitert.

Wie wir gesehen haben, bedingt die JITProduktionsphilosophie - Flexibilität im Bereich der Fertigung - drei aufschaukelnde Kreislaufbeziehungen (in Fig. 9 grün dargestellt) mit entsprechender Kostenfolge im Bereich Personal, Anlagen und Rohstofflager, während die traditionelle Produktionsstrategie - hohe Auslastung, Abdeckung der Bedarfsschwankungen aus Lager - nur eine aufschaukelnde Kreislaufbeziehung im Bereich des Fertiglagers (in Fig. 9 gelb dargestellt) mit entsprechender Kostenfolge bewirkt. Je nach dem Gewicht der Kostenelemente wird man sich für die eine oder andere Produktionsstrategie entscheiden.

\section{Beispiele aus dem Bereich der chem. Wirkstoffherstellung}

\subsection{Bemerkungen zur chemischen Wirkstoffherstellung}

Typisch für die chemische Wirkstoffherstellung sind folgende Gegebenheiten:

- Herstellung in Mehrprodukteanlagen (Mehrzweckanlagen)

- Kampagnenbetrieb

- mehrstufige Synthesen

- Vielzahl von Produkten

- kleine Mengen

- hohe Wertschöpfung (wertvolle Produkte)

- schwankende Bedarfe

- Koordinationsprobleme (Auslastung der Anlagen)

- hohe Anforderungen an Sicherheit, Qualität und Personal

- personalintensiv

Die organisatorische Einheit für die Fertigung ist der 'Betrieb'. In der Regel hat ein solcher Betrieb nachstehende Struktur:

- Betriebsleiter (Chemiker)

- Betriebslabor (Laboranten, Laborgehilfen)

- Betrieb:

Kader (Meister, Vorarbeiter)

Betriebspersonal (Chemikanten, Operators)

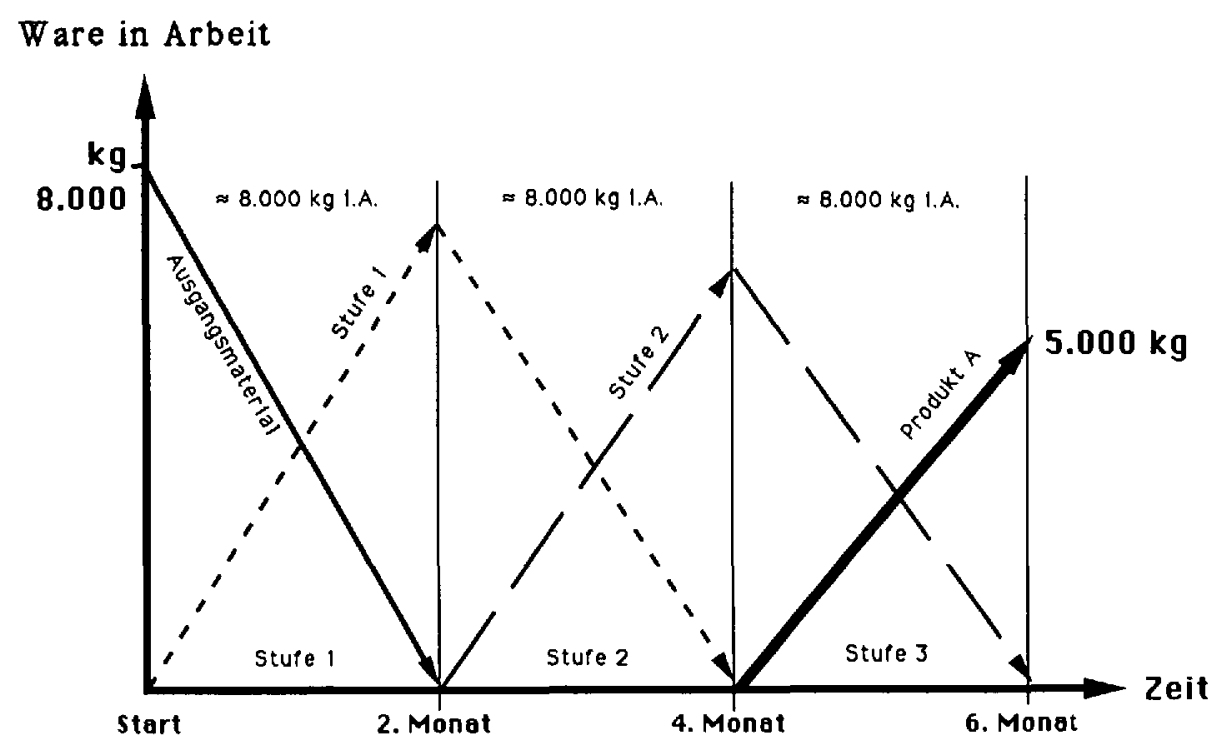

Fig. 5. Materialfluss Kampagnenbetrieb. Gewichtsausbeute pro Stufe $85 \%$. 


\subsection{Beispiel 'Kleinbetrieb'}

\subsubsection{Problemstellung}

Anhand eines Kleinbetriebes, welcher angenommenerweise zwei Verkaufsprodukte in seinem Produktionsprogramm führt, soll untersucht werden, ob es für das Unternehmen wirtschaftlicher ist, die Produkte nach der modernen JIT-Philosophie oder nach der traditionellen 'Anlagenbewirtschaftungsmethode' herzustellen. Um eine klare Antwort auf obige Frage zu erhalten, werden die beiden Szenarien absichtlich extrem in der einen oder in der anderen Richtung abgehandelt.

Annahmen (für beide Szenarien gleich):

- Anzahl Verkaufsprodukte

- Anzahl Fertigungsstufen pro Produkt

Total Fertigungsstufen

- Mittlerer Jahresbedarf Produkt A

Produkt B

- Gewichtsausbeute pro Fertigungsstufe

- Wert Ausgangsmaterialien (bzw. Zwischenprodukt)

- Wert Produkte (Herstellungskosten)

- Kosten einer Mehrzweckanlage (exklusive Bauten)

- Lebensdauer MZ-Anlage (Abschreibungsdauer)

- Unterhaltskosten Anlagen

- Kapitalkosten

- Lagerkosten, 10\% vom Wert Zwischenprodukte pro Jahr Endprodukte pro Jahr

- Personalkosten

2

3

6

$5000 \mathrm{~kg}$

$5000 \mathrm{~kg}$

$85 \%$

$150 \mathrm{Fr} . / \mathrm{kg}$

$500 \mathrm{Fr} . / \mathrm{kg}$

\subsubsection{Szenario I: Extreme Anlagen- bzw.}

Personalbewirtschaftung

Strategie:

- wenig Anlagen, hohe Auslastung

- Bedarfsschwankungen werden aus Lagerbeständen abgedeckt (Flexibilität durch Lager)

Lösung:

- Herstellung des Jahresbedarfs in 2 Kampagnen

1. Halbjahr Produkt A ( $3 \times 2$ Monate)

2. Halbjahr Produkt B ( $3 \times 2$ Monate)

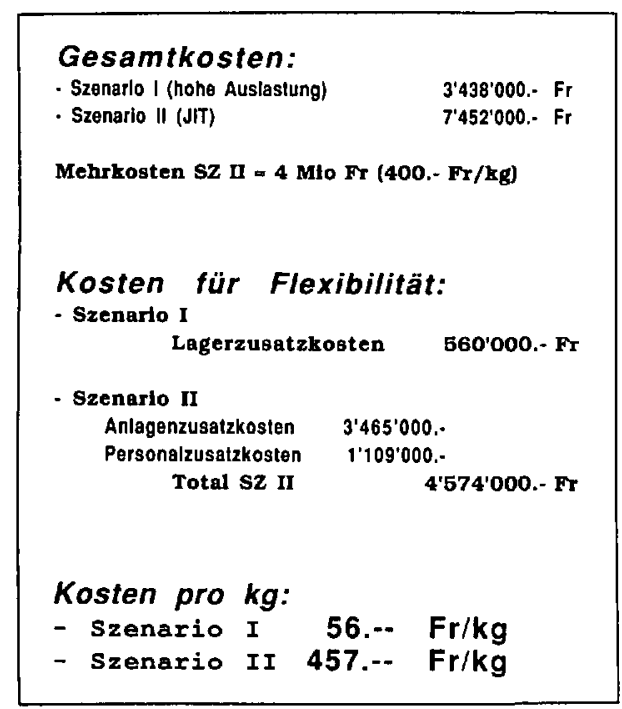

Fig. 6. Vergleich der Szenarien I/II

\section{Kosten für Anlagen}

Unterhalt der Anlagen [7 Mio. $\times 3 \%$ ]

Total Anlagen
7 Mio. Fr.

10 Jahre

$3 \%$ der Anlagekosten $7 \%$

$15 \mathrm{Fr} . / \mathrm{kg}$

$50 \mathrm{Fr} . / \mathrm{kg}$

$45 \mathrm{Fr} . / \mathrm{h}$

Bemerkung zur Abwicklung und Lagerhaltung. In Fig. 5 ist die Produktionsabwicklung mit den entsprechenden Lagerbewegungen (Ware in Arbeit) für ein Produkt dargestellt. Es wird angenommen, dass die mittlere Gewichtsausbeute pro Stufe $85 \%$ beträgt. Für die Herstellung von $5000 \mathrm{~kg}$ Produkt über 3 Fertigungsstufen werden also $c a .8000 \mathrm{~kg}$ Ausgangsmaterial benötigt. Einfachheitshalber wird angenommen, dass während der ganzen Herstellperiode eines Produktes sich durchschnittlich $8000 \mathrm{~kg}$ Ware in Form von Ausgangsmaterial bzw. Zwischenprodukt in Arbeit befindet, obwohl die zu lagernde Menge von Fertigungsstufe zu Fertigungsstufe abnimmt.

Kosten für das Szenario I [3]:

\section{Lagerkosten}

Sicherheitsbestand Prod. A, B $[2 \times 2500 \mathrm{~kg} \times 500 \mathrm{Fr} . \times 10 \%]$

Arbeitslager Prod. A, B $[2 \times 2500 \mathrm{~kg} \times 500 \mathrm{Fr} . \times 10 \%]$

Ware in Arbeit $[2 \times(8000 \mathrm{~kg} \times 0,5 \times 150 \mathrm{Fr} . \times 10 \%)]$

$$
\text { Total Lager }
$$

\section{Bemerkungen zur Abwicklung und}

Lagerhaltung

- Es werden nur die für den Vergleich der Szenarien relevanten Kosten ( $=$ strategische Kosten) ausgewiesen.

- Unter der Position Lagerkosten erscheint neben den bisher erwähnten Aufwendungen für die Haltung eines Sicherheitsbestandes und die Lagerung der 'Ware in Arbeit' noch ein Betrag für ein 'Arbeitslager der Produkte'. Es wurde die für das Szenario I ungünstige Annahme getroffen, dass neben dem Sicherheitslager als Folge der kampagnenweisen Herstellung im Mittel zusätzlich ein halber Jahresbedarf der Produkte an Lager gehalten wird.

5.2.3. Szenario II: Extreme JIT-Produktion

Das obige Unternehmen entschliesst sich für die JIT-Produktionsphilosophie.

\section{Strategie}

- minimale Lagerbestände

- Produktion auf Abruf

- Gewährleistung der Lieferbereitschaft durch Flexibilität in der Produktion

$250000 \mathrm{Fr}$ $250000 \mathrm{Fr}$ $120000 \mathrm{Fr}$

$620000 \mathrm{Fr}$.

Abschreibung 1 MZ-Anlage [7 Mio./10 Jahre]

Kosten für gebundenes Kapital [ 7 Mio. $\times 0,5 \times 7 \%$ ]

3. Personalkosten [21 Pers. $\times 11$ Mon. $\times 160 \mathrm{~h} \times 45 \mathrm{Fr}$.] 


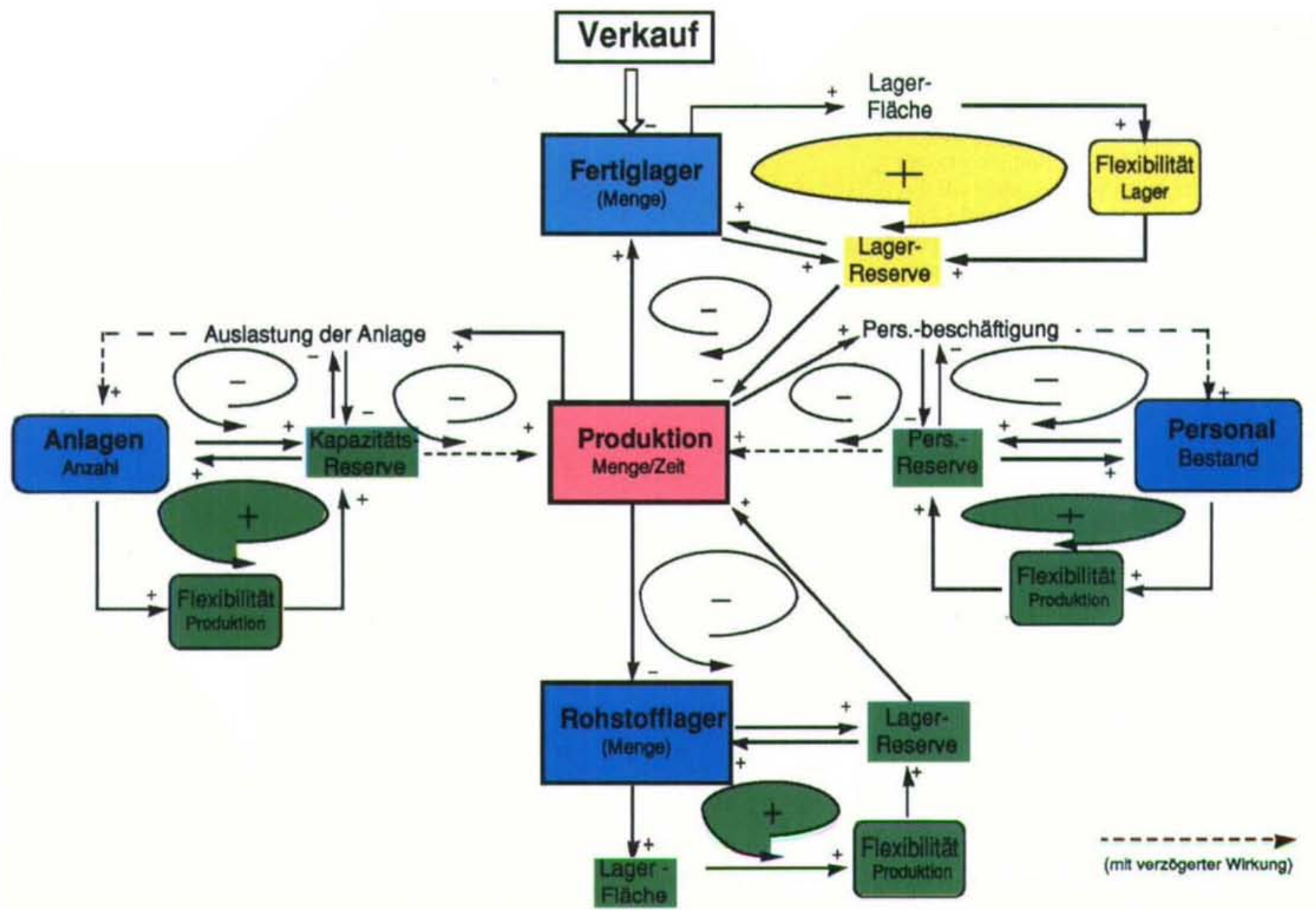

Fig. 8. Erweitertes Produktionsvernetzungsmodell. Flexibilität durch Lager (gelb) oder Produktion (grün).

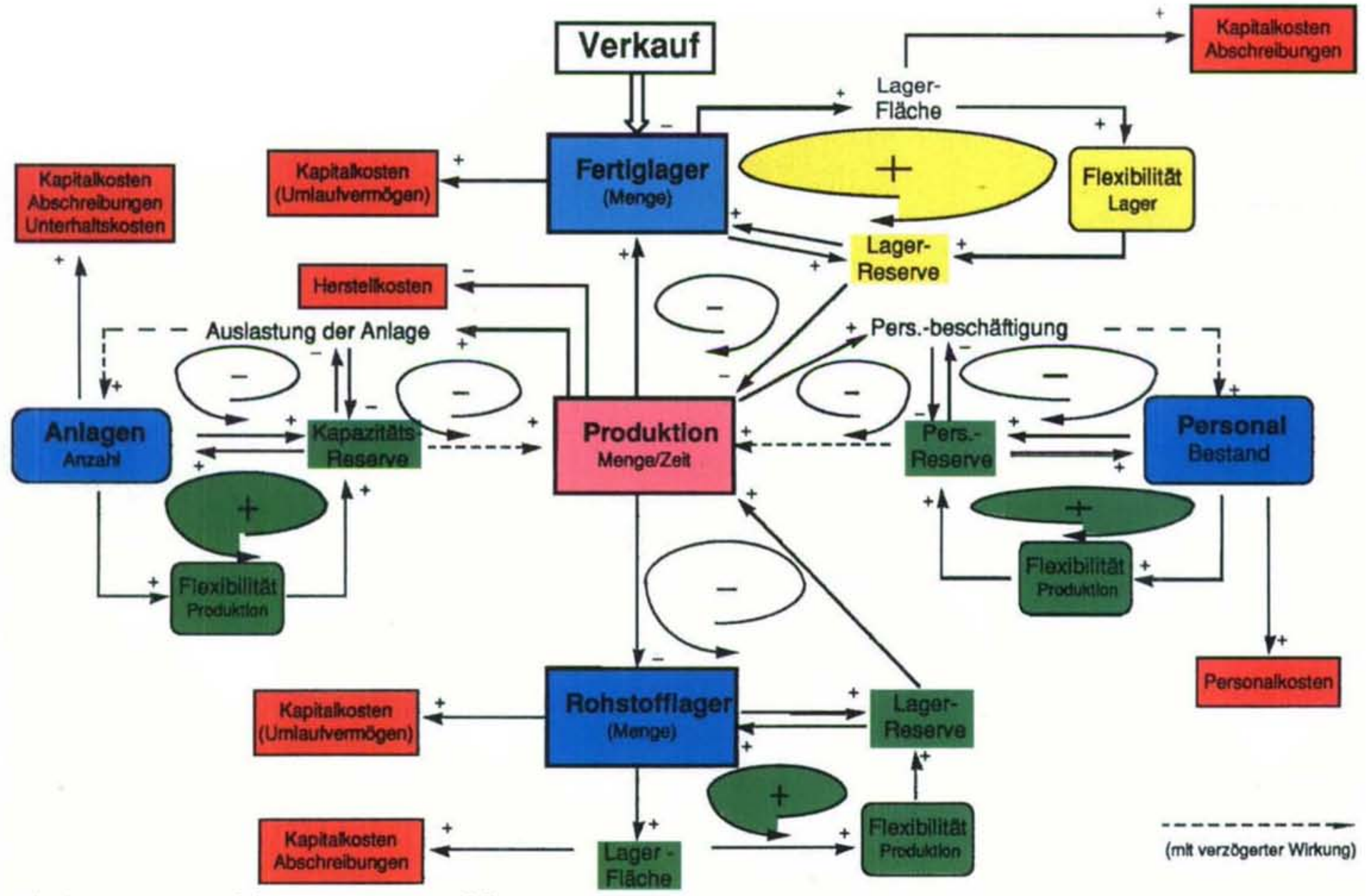

Fig. 9. Integrales Produktionsvernetzungsmodell 
Annahme

- gleiches Unternehmen, gleiches Produktionsvolumen wie im Szenario I

- Bedarf für Produkt A und B fällt gleichzeitig an

Lösung

- Produkte A und B werden gleichzeitig hergestellt (Parallelproduktion)

- Die Durchlaufzeiten werden pro Produkt von 6 auf 3 Monate reduziert

Notwendige Mittel

- $4 \mathrm{MZ}$-Anlagen

- 2 reduzierte Betriebsmannschaften $(1,7 \times$ SZ I): 35 Personen

Bemerkungen zur Lagerhaltung. Da die Wiederbeschaffungszeit nur 3 Monate beträgt, kann auf eine spezielle Lagerhaltung bei den Produkten verzichtet werden. Obwohl als Folge der flexiblen Produktionsphilosophie ein grösseres Rohstofflager gehalten werden müsste, wird davon ausgegangen, dass keine zusätzlichen Kosten in diesem Bereich anfallen.

Kosten für das Szenario II:

1. Lagerkosten

Sicherheitsbestand Prod. A, B

Arbeitslager Prod. A, B

Ware in Arbeit $[2 \times(8000 \mathrm{~kg} \times 0,25 \times 150 \mathrm{Fr} . \times 10 \%)]$

Total Lager

2. Kosten für Anlagen

Abschreibung $4 \mathrm{MZ}$-Anlagen $[4 \times \mathrm{SZ} \mathrm{I]}$

Kosten für gebundenes Kapital [28 Mio, $\times 0,5 \times 7 \%$ ]

Unterhalt der Anlagen [28 Mio $\times 3 \%$ ]

Total Anlagen

$60000 \mathrm{Fr}$

$2800000 \mathrm{Fr}$ $980000 \mathrm{Fr}$ $840000 \mathrm{Fr}$

$4620000 \mathrm{Fr}$.

3. Personalkosten [35 Pers. $\times 11$ Mon. [4] $\times 160 \mathrm{~h} \times 45$ Fr.]

Total Szenario II

$2772000 \mathrm{Fr}$.

$7452000 \mathrm{Fr}$

\subsubsection{Diskussion der Resultate}

Der Vergleich der Szenarien (s. Fig. 6) ergibt, dass in unserem Beispiel die 'JIT. Produktionsstrategie' offensichtlich die falsche Strategie ist. Es scheint wirtschaftlicher zu sein, die (teuren) Anlagen optimal zu nutzen und die Bedarfsschwankungen aus Lagerbeständen aufzufangen. Im Vergleich zu den übrigen strategischen Kosten sind die in diesem Beispiel (SZ I) bewusst hoch angesetzten Lagerkosten vergleichsweise bescheiden. Selbst wenn man davon ausgeht, dass im Szenario II nach Abschluss des Produktionsprogramms das Personal anderweitig eingesetzt werden kann, ist das Szenario II um ca. 2 Mio. Fr. pro Jahr teurer! Auch der Einsatz einer zweiten MZ-Anlage zur Verkürzung der Durchlaufzeiten und damit zur Reduktion der Lagerkosten im Szenario IB scheint nicht wirtschaftlich zu sein (s. Fig. 7).

\subsection{Serie- oder Parallelproduktion als Strategie}

Bei der Herstellung eines Produktes, welches über mehrere Stufen erfolgt, ste-

\section{Produktionsvorschlag}

\begin{tabular}{llllr}
\hline $\begin{array}{l}\text { Produk- } \\
\text { tion }\end{array}$ & Dauer & Menge & $\begin{array}{l}\text { Herstel- } \\
\text { lungs- } \\
\text { kosten } \\
{[\mathrm{Fr} / \mathrm{kg}]}\end{array}$ & $\begin{array}{c}\text { Lager- } \\
\text { kosten }\end{array}$ \\
\hline [Woche] $]$ & {$[\mathrm{kg}]$} & {$[5]$} \\
\hline 1. Stufe & 10 & 24500 & 58 & 27300 \\
2. Stufe & 10 & 28700 & 63 & 62600 \\
3. Stufe & 26 & 50000 & 65 & 243750 \\
\hline Total & 46 & 50000 & 65 & 333650
\end{tabular}

Frage: Ist die Parallelproduktion zur Reduktion der Lagerkosten sinnvoll?

Die Antwort ist einfach, wenn man berücksichtigt, dass die jährlichen Kapitalkosten für eine einzige zusätzliche MZ-Anlage 1,1 Mio. Fr. ausmachen (s. 5.2.2), die maximal möglichen Einsparungen aber nur 0,3 Mio. Fr./a betragen. Wie im vorangegangenen Beispiel ergibt eine einfache Investitionsrechnung, dass die Anschaffung [6] auch nur einer zusätzlichen MZAnlage zur Reduktion der Lagerkosten bei weitem nicht lohnend ist (Invest $7 \mathrm{Mio}$, Einsparung 0,33 Mio./a, Laufzeit 15 Jahre $\rightarrow$ DCFR $=-3,9 \%$, NPV bei $7 \%$ Verzinsung -4,0 Mio. Fr.).

\subsection{Beispiel Losgrösse}

In der Produktion stellt sich immer wieder die Frage nach der optimalen 'Losgrösse'. Obwohl die modernen Produktionsstrategien (JIT) immer kleinere 'Losgrössen' und damit auch kleinere Fertigungseinheiten (Apparaturen) fordern, weiss man aus Untersuchungen, dass mit jeder Verdoppelung der Produktionsmenge (Losgrösse?), die Produktionskosten potentiell um 20-30\% gesenkt werden können (Erfahrungskurvenkonzept; Economy of Scale). An einem Beispiel aus der Wirkstoffherstellung soll dieser Sachverhalt überprüft werden.

Problemstellung: Produktionsstudie zur Herstellung von $200 \mathrm{~kg}$ bzw. $2000 \mathrm{~kg}$ eines Produktes.

Merke: Es werden der Produktionsmenge angepasste Lösungen ausgearbeitet.

\section{Lösung:}

\begin{tabular}{lcc}
\hline & $\begin{array}{c}\text { Variante A } \\
{[200 \mathrm{~kg}]}\end{array}$ & $\begin{array}{c}\text { Variante B } \\
{[2000 \mathrm{~kg}]}\end{array}$ \\
\hline Apparategrösse [1] & 630 & 10000 \\
Kapazität [kg/Woche] & 30 & 300 \\
Produktionsdauer [Woche] & 7 & 7 \\
Arbeitsaufwand [h/kg] & 7.5 & 1 \\
Kosten & & 75 \\
Rohstoffe [Fr. $/ \mathrm{kg}]$ & 300 & 40 \\
Personal [Fr./kg] & 675 & 85 \\
Rüstkosten [Fr./kg] & \\
$\quad$ Variante A: $135000 \mathrm{Fr}$. & & \\
Variante B: $170000 \mathrm{Fr}$. & & \\
Total & \\
Herstellungskosten [Fr. $/ \mathrm{kg}]$ & 1050 & 200
\end{tabular}

Resultat: Verbilligung durch Produktion 'grosser Lose' Fr. 850/kg!

Das produktionsstrategische Ziel müsste folglich heissen: Herstellung möglichst grosser Lose in möglichst grossen Apparaturen!

Das Ausmass der Verbilligung entspricht der Erwartung gemäss des Erfahrungskurvenkonzepts (ca. 30\% Verbilligung pro Verdoppelung der Losgrösse). In diesen Überlegungen sind die sogenannten strategischen Produktionskosten nicht berücksichtigt (bes. Anlagekosten). Es ist aber zu berücksichtigen, dass die Apparategrösse bei den Anlagekosten eher eine untergeordnete Rolle spielt (Anteil der Apparatekosten an den Gesamtkosten $<1 / 3$, davon geht wiederum nur ein kleiner Teil zu Lasten der Grösse der Apparatur).

\section{Schlussbemerkungen}

Mit diesen Ausführungen wurde versucht, die Problematik um die Produktionsstrategien im Bereich der chemischen Wirkstoffherstellung etwas auszuleuchten. Wie wir gesehen haben, sind die Zusammenhänge recht komplex. Es ist kaum möglich, auf diese vielschichtigen Probleme eine einfache Antwort zu geben. Ein möglicher Lösungsansatz besteht darin, dass man versucht, die Zusammenhänge umfassend darzustellen und die Auswirkungen im Unternehmen ganzheitlich zu 
erfassen. Die aufgeführten Beispiele haben gezeigt, dass man unter Umständen zu falschen Schlüssen gelangt, wenn man sich bei der Suche nach Lösungen auf Teilaspekte beschränkt. Eine allgemein gültige Lehrbuch-Produktionsstrategie wird es auch in der Zukunft kaum geben. In jedem einzelnen Fall wird man überlegen müssen, was die richtige Antwort in der speziellen Situation ist.

[1] Zins auf Anlagevermögen; über die Lebensdauer einer Anlage (kalkulatorische Abschreibungsdauer) ist im Mittel die Hälfte des Anschaffungswertes in der Anlage gebunden.

2] Es wird in 2 Schichten à 5 Tage/Woche gearbeitet.

[3] Jahreskosten.

[4] Personal muss für das ganze Jahr bezahlt werden (Flexibilität bedingt Halten von Reserven!).

[5] Nur Arbeitslager; gleichmässiger Abgang des Produktes wurde angenommen.

[6] Einsatz bedeutet strategisch gesehen Anschaffung!

\title{
MRPII Implementation in the Process Industry
}

\author{
Robert Russell*
}

The objective of my presentation is to describe to you our experience of a Manufacturing Resource Planning, (MRPII) Implementation within our Dyestuffs \& Chemicals manufacturing site. We saw this as an opportunity to integrate our approach, not only within the site, but also with our customers and suppliers. We also saw it as a natural progression, building on the successes of our Total Quality Management (TQM) Process which had already begun to challenge many of our traditional attitudes and assumptions.

My presentation will be in 3 main parts:

- The Project

- Our experiences, lessons and conclusions

- Our future expectations

But firstly, let me give a little background about how the Clayton Aniline Company $(C A C)$ fits into the Ciba-Geigy Group.

$C A C$ is a mono-divisional chemical manufacturing site acting as one of the major international supply points for the Ciba-Geigy Dyestuffs and Chemicals Division worldwide. The manufacturing is supported on site by other functions such as Research \& Development, and Engineering. Manufacturing demand is received from the Division Logistics Department in Basle and production of finished dyestuffs and some intermediates is shipped back to Basle for subsequent manufacture or distribution, by Logistics, to worldwide selling companies. There is therefore no sales and marketing at $C A C$.

We are a batch manufacturer with a mixture of street based and multi-purpose plant. We are based in the centre of Manchester, a major city in the northern part of England. The site has been manufacturing dyestuffs for over 100 years and had a traditional culture with many information levels.

\section{The Project}

The goals of the project were clearly defined:

To support the $C A C$ strategic objectives of improving:

- Delivery reliability

- Manufacturing flexibility

- Cost effectiveness

and support, in the longer term, moves towards a J.I.T. philosophy.

The original concept was of a modernisation of the manufacturing support computer systems, but this was rapidly extended to a fundamental review and challenge to the traditional organisation, responsibilities and, in particular, performance measurements. This wider concept of integration was building on the review of roles and responsibilities which had been initiated by the TQM education. The importance of an integrated approach to performance measurement was rapidly appreciated, and forms a continuing theme throughout this presentation.

The concept of integration as a collective responsibility of the people working in the business and covering areas of:

- Customer/Supplier Relationships

- Business Goals and Targets

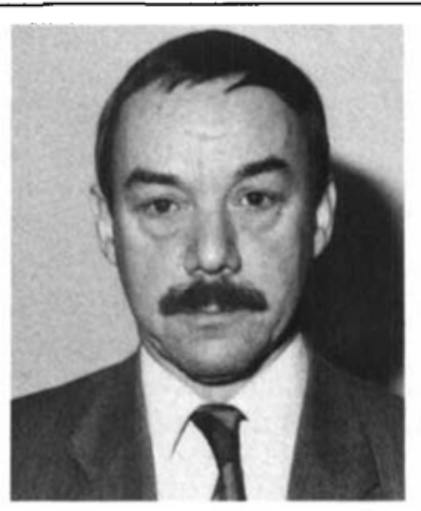

Bob Russell was born in England and gained a degree in Electronic Engineering in 1966. Following this, he worked for a number of U.K. Electronics companies and was involved in the development of aircraft communications equipment and training simulators for the military services. In $1973 \mathrm{hc}$ qualified as a Chartered Accountant gaining general finance and administrative experience through the audit of a number of U.K. multi-national companies. Joining Ciba-Geigy in 1974, he gained further Systems Analysis and software development experience through his involvement in the development and implementation of U.K. Group Finance Systems. In 1985 he moved to his current position, as Director of Finance with The Clayton Aniline Company, a dyestuffs manufacturing Company within the Ciba-Geigy Group. Here he was responsible for the implementation of the Company's MRPII Project. His interests relate to his broad technical and administrative experience, and cover the management of change through Total Quality Management initiatives, and particularly the impact of Performance Measurement on the roles and responsibilities of individuals and the need to integrate these measurements with the overall business strategy.

and supported by an emphasis on formality of process and information flow, was a major part of the education program. The need for a formal, integrated target setting and performance measurement system which drives the integration concept was also emphasised.

The MRPII methodology and project itself was divided into 5 main phases:

- Education

- Data reviews

* Correspondence: R. Russell

The Clayton Aniline Company

P.O.Box 2

Ashton New Road

Clayton

Manchester M114AP 